Página inicial: 193 - Página final: 213

Tipo de artículo: de revisión

\title{
Posturas en la atención psicosocial a víctimas del conflicto armado en Colombia ${ }^{3}$.
}

Postures in the psychosocial care to victims of the armed conflict in Colombia.

Recibido: julio de 2015 Revisado: octubre de 2015 Aceptado: noviembre 20 de 2015

Por: Manuel Alejandro Moreno Camacho ${ }^{1}$, Maria Elena Díaz Rico².

\section{Resumen.}

El artículo presenta una revisión de la producción académica nacional relacionada con el tema de la atención psicosocial a víctimas del conflicto armado. La revisión se realizó a partir del año 2000, con el objetivo de situar las principales consideraciones sobre los procesos de acompañamiento psicosocial a la población víctima de la violencia por el conflicto armado en la historia reciente de Colombia. El análisis se hizo a partir de cuatro categorias: 1) Iniciativas estatales para la atención psicosocial a víctimas del conflicto armado; 2) Reflexiones investigativas sobre procesos de atención a víctimas; 3) Recomendaciones para las instituciones y profesionales que adelantan procesos de atención y reparación integral a víctimas; y 4) La reivindicación del sujeto en los procesos de atención. Los resultados de este trabajo presentan un panorama sobre las principales posturas asumidas en los escenarios de atención psicosocial a víctimas del conflicto en Colombia.

\section{Palabras clave.}

Atención psicosocial, Enfoque psicosocial, Víctimas, Conflicto armado.

\section{Abstract.}

The article presents a review of the national academic production related to the subject of psycho-social care to armed conflict victims. The review was conducted from the year 2000 , in order to locate main considerations about the processes of psycho-social accompaniment to the victims of violence by the armed conflict in the recent history of Colombia. The analysis was made from four categories: 1) State initiatives for psycho-social care to victims of the armed conflict; (2) research reflections on processes of care to victims; (3) recommendations for the institutions and professionals carried out processes of care and full reparation to victims; and (4) the claim of the subject in the care processes. The results of this work present an overview of the main positions assumed in the scenarios of psycho-social care to victims of the conflict in Colombia.

\section{Key words.}

Psycho-social care; Psycho-social approach; Victims; and Armed conflict.

\footnotetext{
${ }^{1}$ Estudiante de Doctorado en Psicología, Universidad del Valle; Psicólogo, Universidad de San Buenaventura Cali; Magister en Sociologia, Universidad del Valle; docente investigador, Instituto de Psicologia; Cali, Colombia. contacto: manalmoreno@yahoo.com

${ }^{2}$ Profesora asociada de la facultad de psicologia, Universidad de San Buenaventura Cali; Psicóloga, Universidad del Valle; Magister en psicologia, Universidad del Valle, Facultad de Psicologia; Cali (Colombia). contacto: mediaz@, usbcali.edu.co.
} 


\section{Introducción.}

La atención psicosocial a víctimas del conflicto armado en Colombia ha sido un proceso en el que han participado instituciones del Estado y organizaciones civiles desde diversas perspectivas y atendiendo a diferentes intereses. La justificación para adelantar acciones dirigidas a mitigar los impactos de la violencia por el conflicto armado en nuestro país es más que evidente. De acuerdo con las cifras oficiales, hasta noviembre de 2014 el número de víctimas registradas era superior a siete millones (Unidad para la atención y reparación integral a las víctimas, 2014a). Esto quiere decir, que el impacto de la violencia ha alcanzado de manera directa a más del 15\% de la población del territorio colombiano. La cifra por sí misma es sorprendente, sin embargo, no logra transmitir la complejidad de la tragedia, pues los efectos de la exposición a los hechos de violencia son tan variables como los sujetos que se han visto sometidos a dichas situaciones.

No es tarea sencilla, entonces, hablar de atención psicosocial para las víctimas de la violencia en Colombia. Los discursos oficiales, así como los de las agencias que invierten recursos para apoyar este proceso, se caracterizan por una pretensión de universalidad utópica que pretende encontrar la fórmula adecuada para mitigar los efectos de la violencia en todos los sujetos que se han visto afectados por ella. Partir de reconocer la complejidad del fenómeno, implica de entrada asumir que tal universalidad no es más que una utopía, un tipo ideal a alcanzar, pero en tanto tipo ideal, dificilmente es posible acceder a él de manera pura en hechos concretos de la realidad social.

La dificultad de este planteamiento radica en que todas las víctimas tienen el mismo derecho a ser reparadas por haber sido sometidas a tales actos de violencia, por lo tanto, desde la perspectiva oficial la política para la atención a víctimas se caracteriza por estar formulada bajo el precepto del para todos, lo que significa para todos por igual. La más reciente norma dirigida a mitigar los impactos de la violencia en Colombia es la ley 1448 de 2011, también conocida como ley de víctimas y restitución de tierras. En un esfuerzo loable y partiendo de la experiencia acumulada en el país en materia de programas de atención a población afectada por el conflicto, esta ley propone de manera explícita que la atención a las víctimas debe adelantarse con enfoque psicosocial. Dicho encargo, implica un gran desafio para las instituciones y profesionales que representan esta perspectiva de trabajo, en la medida en que tiene la apariencia de ser la fórmula adecuada para aliviar el sufrimiento de los afectados por el conflicto.

No obstante, uno de los principales cuestionamientos que se suscitaron a partir de este planteamiento ha sido la pregunta por ¿Qué es lo psicosocial? o ¿Qué es el enfoque psicosocial?, pues pareciera que la incorporación de este componente en la ley hubiese estado alentado por el uso cotidiano de un término que se ha hecho muy popular en los programas de atención dirigidos a poblaciones en situación de vulneración o riesgo de vulneración de sus derechos. Y es que en el contexto de las intervenciones sociales es común encontrar el uso de la fórmula atención psicosocial para designar prácticas de diversa índole asociadas más al conjunto de profesionales que las ejecutan, que a una manera de proceder cimentada en los principios que la orientan. La denominación atención psicosocial se usa para referirse a las acciones desempeñadas por quienes conforman los equipos psicosociales -generalmente profesionales de la salud mental y de las ciencias sociales-, 
como si el hecho de obrar en nombre de una profesión o disciplina fuese suficiente para considerar que dichas acciones están basadas en principios conceptuales, metodológicos y éticos orientados por una perspectiva psicosocial (Moreno \& Moncayo, 2015; Villa, 2012).

De acuerdo con esta discusión, en la Facultad de psicología de la Universidad de San Buenaventura Cali avanzamos en la investigación Posturas y prospectiva en el abordaje psicosocial de la población víctima de la violencia por el conflicto armado. En el marco de dicho proceso de investigación, el presente escrito se ocupa de una revisión documental de la producción académica nacional relacionada con el tema de la atención psicosocial a víctimas del conflicto armado. La revisión se realizó con algunos documentos publicados a partir del año 2000, con la intención de situar las principales consideraciones sobre los procesos de acompañamiento psicosocial a la población víctima de la violencia por el conflicto armado en la historia reciente de Colombia. El análisis de los documentos condujo a la reflexión a partir de tres categorias: 1) Iniciativas estatales para la atención psicosocial a víctimas del conflicto armado; 2) Reflexiones investigativas sobre procesos de atención a víctimas; 3) Recomendaciones para las instituciones y profesionales que adelantan procesos de atención y reparación integral a víctimas; y 4) La reivindicación del sujeto en los procesos de atención. Los resultados de este trabajo presentan un panorama sobre las principales posturas asumidas en los escenarios de atención psicosocial a víctimas del conflicto en Colombia.

\section{Iniciativas estatales para la atención psicosocial a victimas del conflicto armado.}

Como señalamos con anterioridad la ley de víctimas y restitución de tierras propone de manera explícita que la atención a las víctimas debe adelantarse con enfoque psicosocial. Es importante entonces remitirnos al discurso oficial y la manera en la que dicho enfoque es definido desde esta instancia. Así, encontramos que la ley de víctimas se refiere a la atención como "la acción de dar información, orientación y acompañamiento jurídico y psicosocial a la víctima, con miras a facilitar el acceso y cualificar el ejercicio de los derechos a la verdad, justicia y reparación" (Colombia, 2012, p.36). En este orden de ideas, lo psicosocial es entendido como uno de los componentes de la atención, específicamente hace parte de las medidas de rehabilitación, sin embargo se advierte que dicho componente deberá ser permanente y transversal durante el proceso de reparación.

De manera más específica, el ministerio de salud y protección social (2013a y 2013b) y la unidad para la atención y reparación integral a víctimas (2014b), se refieren a los fundamentos del enfoque psicosocial para señalar sus principales características. Entre ellas se destaca que el enfoque psicosocial es ubicado como el resultado de la experiencia de acompañamiento a poblaciones afectadas por eventos generados en contextos de violencia sociopolitica, particularmente en el contexto latinoamericano. De esta manera, el enfoque está orientado al restablecimiento de los derechos vulnerados y la reivindicación de la dignidad de los sujetos afectados por los hechos de victimización.

Para avanzar en ese sentido, el enfoque psicosocial se orienta por una apuesta ética en la relación con las víctimas que se fundamenta en el reconocimiento de la capacidad de agencia y la despatologización del sufrimiento. Esta apuesta es presentada por la unidad para la atención y reparación integral a víctimas a partir de tres premisas. La primera premisa está 
orientada a contemplar que las expresiones de sufrimiento han sobrevenido como respuestas normales frente a los eventos anormales propiciados por los hechos violentos en el marco del conflicto. Esta premisa invita a pensar que las causas estructurales del sufrimiento de las víctimas no son estrictamente intra-psíquicas, sino que resultan de las relaciones establecidas en los contextos de desarrollo y la exposición a los eventos de violencia, por lo tanto, es necesario considerar las características contextuales para entender el sufrimiento de los afectados, más allá de categorizar o diagnosticar a partir de criterios estandarizados. La segunda premisa, en estrecha relación con la anterior, se refiere al reconocimiento de los recursos propios con los que cuentan los sujetos y las comunidades afectadas por hechos de victimización, incluso más allá del sufrimiento provocado por las acciones violentas. Es decir, se trata de considerar a las víctimas como sujetos que no solamente están sufriendo, sino que cuentan con recursos para afrontar su situación actual de vida, lo que implica también reconocer que los hechos de victimización no son el referente estructural que define su situación actual, sino que tienen el valor de variables en una cadena de acontecimientos históricos que constituyen la vida de un sujeto o una comunidad. Por su parte, la tercera premisa está fundamentada en el enfoque psicosocial de dignificación y reconocimiento, y sienta las bases para el principio de corresponsabilidad en el marco de los procesos de atención a las víctimas. Se trata del reconocimiento de que "todas las personas pueden hacer algo frente al sufrimiento de los otros" (Unidad para las víctimas, 2014b, p.11), es así como, las acciones dirigidas a los sujetos afectados por hechos de victimización tienen el carácter de reivindicar la dignidad que les fue arrebatada por las acciones de violencia.

De esta manera, la postura oficial para definir el enfoque psicosocial de cuenta de la inscripción en una tradición y experiencia que se caracteriza por el reconocimiento de la victimización en el marco del conflicto armado como un hecho social que requiere de un análisis que abarqué la complejidad implicada en el mismo. También da cuenta de la incorporación de marcos de referencia que se ubican desde una perspectiva crítica frente al discurso hegemónico de la salud mental y la clasificación psicopatológica, avanzando hacía una perspectiva inspirada en los planteamientos de la psicología social y la psicología comunitaria desarrolladas principalmente en contextos latinoamericanos. Estos planteamientos se ven refrendados por las reflexiones investigativas que se presentan a continuación.

\section{Reflexiones investigativas sobre las prácticas de atención a victimas del conflicto armado.}

Los profesionales que ejecutan las intervenciones y reflexionan sobre sus experiencias, así como los investigadores que han revisado las que han sido realizadas por otros, sugieren puntos sensibles a tener en cuenta que están orientados a favorecer la calidad y pertinencia de los procesos. Entre los puntos más significativos de este tipo de reflexiones se encuentran preguntas como: ¿Cuál es el objetivo de la atención? ¿Cuáles aspectos incluye y como todos intervienen en el resultado que se obtiene al final? La lectura de los documentos permite acercarse a algunas posturas en la forma como se ejecutan las intervenciones, así como también al señalamiento de algunos vacíos en su desarrollo. En el caso de las posturas asumidas, se destaca que en ocasiones los mismos ejecutores no son conscientes de los efectos que generan las decisiones que toman al optar por una u otra manera de proceder; mientras que en el segundo aspecto, se muestran campos que requieren ser abordados para aportar conocimiento, de tal forma que el proceso de atención cada vez logre mejores 
resultados para el bienestar de la población afectada, así como también para el interés general de nuestro país.

En las intervenciones revisadas se hace evidente una tendencia hacia perspectivas que abogan por promover las capacidades de los sujetos y potenciar sus recursos para enfrentar las situaciones. Estas perspectivas se posicionan como una respuesta frente al análisis de experiencias centradas en posturas asistencialistas que perpetúan a las personas en el lugar de víctimas. Perspectivas como las de Estrada, Ibarra \& Sarmiento (2003), MartínBeristain (2004, 2012), Arias, Arévalo \& Ruíz (2002), Arévalo (2010), Estrada, Ripoll \& Rodríguez (2010), Wilches (2010), Rebolledo y Rondón (2010), Grupo de psicología social crítica (2010a, 2010b), Duque \& Gordon (2012), Moreno (2013) y Moreno \& Moncayo (2015), enfatizan en la necesidad de evitar narrativas que refuercen la posición de víctima e incentivan unas más agenciadoras para enfrentar las situaciones. A partir de allí, se propende por el reconocimiento de habilidades y recursos de los sujetos y comunidades, con miras a una posible resignificación de las experiencias que esté orientada a la asunción de una posición de control frente a la vida, justamente para hacer frente a aquello que se vulnera por el hecho de haber sido sometidos a hechos victimizantes.

En este contexto de análisis se presentan dos vertientes para el acompañamiento psicosocial. Por un lado están las estrategias colectivas de trabajo, en las que se privilegia la constitución de grupos y el rescate de valores comunitarios, es decir, se propende por el fortalecimiento de recursos de afrontamiento desde la perspectiva relacional. Por otro lado, las explicaciones se cargan a los análisis de los recursos individuales para el afrontamiento de las situaciones que genera la victimización.

Mc. Cauley (2002) por ejemplo, critica las intervenciones centradas en aspectos psicológicos, que desconocen el hecho de que las problemáticas se vivencian colectivamente y por lo tanto, requieren una intervención comunitaria. A su vez, Martín-Beristain (2012) enfatiza en la necesidad de enfoques que no descontextualicen las respuestas o problemas psicológicos y permitan entender las reacciones de las víctimas según el contexto en el que se presentan. El mismo Martín-Beristain (2004), propone abordar la intervención psicosocial desde una perspectiva holística que concibe al ser humano como un sistema en el que se relacionan sus sentimientos, emociones, cuerpo y espiritualidad, apostándole a una intervención que toque cada una de estas áreas, pues considera que al dirigir el trabajo solamente hacia una no se logra la meta del bienestar. Estos autores recomiendan moverse hacia un enfoque basado en la comunidad, que tenga en consideración sus fortalezas y riquezas culturales, desde el rescate y fortalecimiento de sus formas organizativas para hacer frente al sufrimiento y para reconstruir entre todos lo que ha sido afectado por los hechos de victimización.

Por su parte, Palacio \& Sabatier (2002), así como Bello (2006a), se inclinan hacía propuestas que consideren la dimensión individual, partiendo de la premisa de que "las maneras de afrontamiento son individuales así las motivaciones sean las mismas" (Bello, 2006a, p.30). Ante esto sugieren que los estudios no se enfoquen únicamente en lo colectivo, sino también en lo individual. Al respecto es posible decir que estas dos posturas no son necesariamente excluyentes, pues las propuestas de trabajo comunitario pueden integrar la perspectiva de fortalecimiento de recursos individuales, lo importante desde la perspectiva psicosocial es el reconocimiento de que el sujeto se forma y transforma en un devenir que es relacional y que 
se caracteriza por ser un espacio de interacciones dialécticas. Así mismo, es relevante tener en consideración la conceptualización acerca del reconocimiento de daños individuales y daños colectivos (Rebolledo \& Rondón, 2010; Novoa, 2013), con el objetivo de construir acciones de respuesta contextualizadas y acordes con las diferentes formas de significación de los eventos de violencia que pueden presentarse en los grupos sociales.

Ahora bien, de acuerdo con los resultados de la revisión realizada se evidencia que la intervención psicosocial en nuestro país ha estado más centrada en la atención de las consecuencias que generan los hechos de violencia y no necesariamente se ha ocupado de la comprensión y desentrañamiento de la estructura del conflicto armado y sus raíces. Cuando el análisis avanza en esa vía, es necesario reconocer las falencias del Estado en el cumplimiento de algunos derechos fundamentales de la población. Estas falencias se hacen evidentes en aspectos como las políticas de cobertura educativa, los servicios de salud y las oportunidades de empleo. Así mismo, es preciso reconocer en la dinámica nacional la lógica de perpetuación de patrones culturales a partir de las prácticas cotidianas que se desarrollan en medio de conflicto.

Considerar las causas estructurales del conflicto y la lógica de relaciones que se constituye a partir de las mismas tiene un efecto sobre las propuestas de atención a la población víctima del conflicto armado, pues ya no se trata exclusivamente de los actores involucrados en la relación de atención, sino que también se consideran diferentes variables que conducen a las situaciones de victimización, que son clave para la comprensión del sufrimiento ocasionado en los sujetos y aportan pistas para avanzar en las posibilidades de transformación. Sin embargo, investigadores como Durán (2002) plantean que en una política pública como la nuestra, centrada en la atención, no hay oportunidad de profundizar en la comprensión de las causas de los problemas y como consecuencia de ello dirigir los esfuerzos a una política que dé prioridad a la prevención.

En este sentido retomamos los señalamientos de Martín-Baró (1998) respecto al quehacer desideologizador que debe cumplir la psicología en contextos de conflicto armado. En lugar de dirigir la atención al ajuste del sujeto al sistema, se trata tambiE us propios intereses yiones. Estas agencias se encargan de avalaritrategias de acompañamiento psicosocial que cuentan én de cuestionar la estructura en la que está inserto y que constituye la base de la problemática social a la que el sujeto ha sido sometido. Al respecto Plata (2006) plantea que uno de los retos que debe enfrentar la investigación social tiene que ver con la pertinencia de las preguntas que como investigadores nos hacemos frente a la problemática ¿Hasta qué punto lo que se está investigando aporta pistas acerca de las raíces del conflicto armado en nuestro país, con el fin de superar las explicaciones que no incluyen todos los elementos de esta compleja realidad?

El incumplimiento de esta función desideologizadora también se puede evidenciar en las restricciones que enfrentan los profesionales a la hora de plasmar por escrito los resultados hallados en sus intervenciones, ya que deben pasar primero por un filtro por parte de las agencias que patrocinan las investigaciones o intervenciones. Estas agencias se encargan de avalar lo politicamente correcto, pues el proceso de revisión y aprobación está mediado por sus propios intereses. En algunos casos la revisión exige que algunos hallazgos y 
sugerencias sean matizados, precisamente los aspectos más interesantes que aportan a la comprensión del fenómeno estudiado (Bello, 2006b).

En esta misma línea de análisis, Toro (2011) presenta un balance sobre algunos programas generados desde del gobierno y otros provenientes de organizaciones no gubernamentales de origen nacional como internacional. En cuanto a los primeros, encuentra como denominador común un mayor énfasis en actividades de tipo asistencialista y de respuesta a las emergencias - lo cual tiene sentido, pues es lo primero que debe garantizar el Estado sin embargo, este marcado énfasis de su intervención deja a la población en una situación flotante respecto al acompañamiento que según la actual ley de víctimas está previsto para la población afectada por el conflicto armado. Esta situación pone en evidencia dos asuntos, por un lado, la necesidad de realizar una intervención integral, es decir, que no se fragmente al sujeto sino que se lo entienda como una totalidad. Desde esta perspectiva la reparación incluye aspectos materiales como las indemnizaciones económicas, así como también emocionales y culturales. Estos aspectos requieren ser integrados en la oferta que brindan las diferentes instituciones para dar una respuesta integral a la problemática. Por otro lado, se señala un cuestionamiento al fortalecimiento de la dependencia de las comunidades hacia los recursos que reciben de parte del Estado, lo cual invita a pensar en la forma cómo se debería entregar estos recursos para que no promuevan la actitud pasiva en la comunidad receptora, de tal forma que los programas estén orientados por un enfoque de acción sin daño.

En esta misma línea de ideas, al preguntarse hacia dónde se dirige la inversión de recursos económicos de las agencias de cooperación internacional, observamos que los recursos se dirigen principalmente a la financiación de programas de atención, más que al desarrollo de procesos de investigación. Esto quiere decir que la inversión que se realiza está dirigida en mayor medida a las consecuencias que deja el conflicto armado, más que a la indagación de las raíces de esta problemática. Es justificable la financiación de programas de atención, pues las necesidades de las poblaciones son amplias y la oferta del Estado no es suficiente para su satisfacción, sin embargo, los procesos de investigación son muy importantes para fundamentar las acciones de atención, más aun cuando la consecuencia de la amplia inversión de recursos en programas de atención es la demanda de resultados para justificar el gasto. A partir de esta lógica resulta muy importante mostrar cifras, por ejemplo, el reporte del número de personas atendidas resulta más importante que el nivel de bienestar integral alcanzado con la atención (Bello, 2006b).

Este es un tema de mucha controversia, pues no se desconoce que las acciones de intervención son de gran importancia para ayudar a mitigar el sufrimiento ocasionado por los hechos de violencia. Sin embargo, la institucionalización de los programas tiene como consecuencia la burocratización de los procesos, de tal forma que la mayoría de las veces los programas se convierten en acciones desgastantes, en las que el diligenciamiento de múltiples formatos resta tiempo para encontrarse, escuchar y trabajar directamente con la población destinataria de los servicios. No obstante, es indiscutible que el seguimiento y la sistematización de los procesos es de gran importancia para la toma de decisiones y para el ajuste de las estrategias de atención. Se trata entonces de encontrar un balance en el que lo más importante sea la calidad de la atención que se brinda a los destinatarios de los servicios. Desafortunadamente, el panorama nacional respecto a este tema deja un 
balance desfavorable, pues la opinión general indica que los procesos administrativos y el levantamiento de evidencia para la presentación de resultados que tengan impacto político se imponen al interés por garantizar un acompañamiento pertinente y respetuoso para con los sujetos a los que está dirigida la atención.

Por su parte, los tiempos dedicados a la intervención también son objeto de discusión, se establece la diferencia existente entre los tiempos institucionales y los tiempos de las comunidades con las que se trabaja, aspecto que incide en el nivel de superficialidad o profundidad con que se identifican las necesidades de las poblaciones destinatarias de los servicios. Es común encontrar que los procesos que se ven atropellados por la lógica de los tiempos institucionales, los cuales que exigen informes con resultados o productos en tiempos que no necesariamente reconocen los tiempos que toma el construir relaciones de confianza con las poblaciones, especialmente cuando han sido víctima de la violencia y la desconfianza es una variable a considerar. En la dinámica de la intervención social es común compartir espacios de la cotidianidad de las poblaciones, los cuales están por fuera de los dispositivos institucionales (Tovar, 2013). Es importante, entonces, considerar el tiempo que toma desentrañar las creencias culturales, traducidas en patrones y prácticas que dotan de sentido el comportamiento de los sujetos y dan pistas para diseñar una intervención (Bello, 2006b; Díaz, Arias, \& Lasso, 2010).

Como consecuencia para el avance del conocimiento a través de la investigación y el bienestar de la población destinataria, se identifica la perdida de esfuerzos debido a que los procesos iniciados se cortan cuando por fin se ha logrado consolidar en los tiempos de la comunidad un vínculo, una credibilidad, y como consecuencia de ello se ha avanzado en la construcción de acciones. En la mayoría de los casos los tiempos institucionales implican que las acciones adelantadas sean suspendidas al acabarse la vigencia de los fondos estatales o el apoyo de las agencias de cooperación, y cuando se vuelve a conseguir una financiación ya algunas comunidades se han dispersado o han cambiado de ubicación territorial, lo cual obliga a empezar nuevamente procesos y esto hace que se pierdan las relaciones que previamente se habian adelantado (Bello, 2006b).

A su vez, los límites de la intervención y las respectivas consideraciones éticas a los que ello conlleva son un campo de reflexión al que los investigadores se dirigen para señalar la urgencia de detener su mirada sobre las implicaciones que trae la atención de la población en contextos de guerra (Grupo de psicología social crítica, 2010b; Moreno, 2013). Los profesionales manifiestan la necesidad de abrir un espacio para hablar y reflexionar con otros colegas acerca de las diversas vicisitudes que deben enfrentar en el ejercicio cotidiano de su labor, sus temores, sentimientos de culpa, duelos, frustraciones; así como discutir preguntas referidas a los límites de su intervención, tales como: ¿Es posible mantenerse al margen de los hechos de agresión que ocurren en su presencia? ¿Cómo asumir la responsabilidad de hacer denuncias ante las instituciones legales correspondientes cuando está en juego la integridad física y emocional de otras personas? Así mismo, se puede poner en riesgo la vida del profesional y este no es un tema que se aborde en los manuales científicos disponibles (Osorio, 2006).

En este orden de ideas, se resalta la necesidad de ocuparse de los que atienden a la población víctima del conflicto. Los profesionales que investigan y atienden esta problemática no 
sólo requieren capacitación conceptual sino también psicológica, orientada a desarrollar competencias para hacer frente a la carga afectiva que acumulan en el ejercicio de su trabajo. En este sentido, se evidencia una necesidad para el avance en la construcción del conocimiento sobre lo psicosocial, dirigida a la atención de los cuidadores y la reflexión sobre el rol asumido por los profesionales que se desempeñan en los programas de atención psicosocial a víctimas del conflicto armado (Díaz, Blandón y Echeverri, 2006; Sánchez y Musitu, 1996).

\section{Recomendaciones para las instituciones y profesionales que adelantan procesos de atención y reparación integral a víctimas.}

Teniendo en cuenta la reflexión que hacen los investigadores sociales y los profesionales que se ocupan de las intervenciones psicosociales con víctimas de la violencia, en este apartado se presentan algunas de las recomendaciones más persistentes para el buen desarrollo de las acciones enmarcadas en procesos de acompañamiento psicosocial. Coincidimos con los autores en la necesidad de diseñar procesos de atención que estén orientados a las diferentes dimensiones de la problemática en lugar de dirigir sus esfuerzos a una sola, ya que el resto de las bases del problema seguirian intactas y no se lograría un efecto positivo a mediano plazo.

Bello (2006a), por ejemplo, propone que la intervención psicosocial incluya elementos que contribuyan a la reconstrucción de la identidad, propiciar la autonomía que permita potencializar sus capacidades de agencia tanto individual como colectiva; favorecer la satisfacción de las necesidades básicas que garanticen su subsistencia, es decir, acciones de protección del Estado; y el despliegue de recursos propios, la activación de redes sociales e institucionales, nuevos lazos y vínculos. En cuanto a la activación de las redes sociales es necesario primero identificar en las que se desenvuelve la vida de los individuos, es decir, su familia, las instituciones educativas, grupos de pares, asociaciones comunitarias, y luego actuar sobre ellas con el objetivo de promover la participación de sus integrantes en la organización de estos grupos, hacer uso de los recursos con los que cuentan para gestionar transformaciones y hacer frente a las problemáticas sociales que enfrentan (San Juan Guillén, 1996; Bello, 2006a; Tovar, 2013). A su vez este trabajo colectivo permite identificar las fortalezas personales que tienen los participantes, gracias a la riqueza cultural de sus lugares de origen, permitiéndoles compartir las prácticas que utilizan para hacer frente al dolor, la incertidumbre y las dificultades en las relaciones familiares y comunitarias. Se recomienda entonces una intervención que identifique los valores, normas, actitudes y expectativas de la población a la que está dirigida, de tal forma que su cosmovisión se vea reflejada en el diseño del proceso de acompañamiento. Así, las estrategias implementadas reflejarán sus actitudes, normas, esperanzas; así como una consideración por aquellas formas de actuar que son de su preferencia y que por tanto facilitan su participación e implicación en los procesos de cambio (Marín, 1996).

Resaltamos la importancia de acercarse a la realidad de ese otro al que se intenta beneficiar para comprender sus formas de relacionarse con los otros, de resolver los problemas, con el fin de producir alternativas que efectivamente se ajusten a lo que cada comunidad específica requiere (Díaz, Arias \& Lasso, 2010). Así mismo, Estrada, Ripoll \& Rodríguez (2010) enfatizan en la necesidad de propiciar espacios de participación de la población 
beneficiaria junto a los equipos psicosociales, de tal forma que se reconozcan las diferentes lecturas de la realidad, se permita ampliar la comprensión y establecer acuerdos para mejorar el proceso de atención. Lo anterior implica asumir un cambio en las relaciones, ya que tradicionalmente las familias son percibidas como usuarias que reciben lo que otro ha diseñado para ellas, en lugar de participar como interlocutoras válidas en el proceso de construcción y afinamiento de la intervención. Tal postura crítica también es compartida por Bello (2006b), quien reconoce el valor del conocimiento compartido en lugar de las soluciones construidas sin el reconocimiento del saber de la comunidad y del investigador. Así mismo, Martín-Beristain (2004) resalta la importancia de permitir la participación de los afectados con sus propuestas, cuestionamientos y trabajo conjunto a la hora de diseñar planes de acción.

Un énfasis que encontramos en esta vía de intervención se dirige a promover acciones que ubiquen a la población beneficiaria en una postura de agencia, en oposición a una postura pasiva. En este sentido se sugiere equilibrar la intervención trabajando tanto en la satisfacción de necesidades y el restablecimiento de derechos, así como también en el fortalecimiento del desarrollo político y comunitario. Si las acciones se centran exclusivamente en los primeros se corre el riesgo de poner a la población en una situación de pasividad a la espera de que sea el Estado quien le resuelva su futuro, excluyéndola de toda responsabilidad, poniéndola en una condición de invalidez e inutilidad, por lo tanto, es importante reconocer la responsabilidad que el Estado tiene en su actual situación, pero también es fundamental identificar cómo se encuentra la comunidad en el momento actual y fomentar la intervención sobre su contexto, con el objetivo de desarrollar en los sujetos un sentido de control y eficacia frente a sus condiciones, que les permita empoderarse y ganar confianza a la hora de enfrentar nuevas situaciones (San Juan Guillén, 1996).

Así las cosas, resaltar la riqueza de los recursos comunitarios ubica a los sujetos posición de agentes y sirve además para reconocer valiosas prácticas para enfrentar el dolor, que deben ser atendidas como guía a seguir con las comunidades (Tovar, 2013). En este sentido, vale la pena reconocer valiosas experiencias comunitarias adelantadas en las que, con sus propios recursos, los sujetos se organizan para hacer frente al dolor. Por ejemplo, experiencias grupales en las que tienen lugar la construcción de nuevas interpretaciones y realidades, a partir de la constituaicón de grupos de soporte en los que los participantes comparten vivencias, emociones y conocimientos, favoreciendo la transformación de su identidad colectiva y a su vez favorecen su reconocimiento como sujetos de derecho y actores políticos (Grupo de psicología social crítica, 2010a). Así mismo, otras experiencias relatadas (Meertens, 2006; Grupo de Jóvenes Afrocolombianos Taller Vida, 2002) resaltan la importancia que tiene propiciar espacios y actividades que permitan a la población reconectarse con aquellos aspectos culturales que aportaban a la definición de su identidad, ya que estos son muy importantes para el fortalecimiento de los recursos de afrontamiento de las comunidades afectadas por la violencia.

Es así como se destaca la necesidad del enfoque diferencial, reclamado por las mismas comunidades que han sido víctimas de los hechos violentos, ya que las necesidades de las distintas poblaciones no son las mismas, la forma como han vivido estos hechos y lo que han perdido es dimensionado de manera diferente dependiendo del grupo étnico, el género, los grupos etarios; por lo tanto se critica el interés de resolver la atención unificándola, 
ya que de esta forma no se da una efectiva reparación a las necesidades de cada grupo o individuo, sino que se hace con base a un grupo ideal que no contempla la forma como se significan los acontecimientos de manera particular (Grupo de jóvenes afrocolombianos - taller vida, 2002; Sacipa, 2013). Así mismo, el enfóque diferencial contribuye a que no se generalice un único modelo para todas las regiones de nuestro país, ya que el conflicto armado no tiene las mismas características en cada región y la forma en que lo asumen sus habitantes también se encuentra atravesada por sus características culturales.

Es importante destacar que entre el conjunto de documentos revisados, una de las estrategias de acompañamiento psicosocial más destacadas es el trabajo de construcción de memoria colectiva (Molina, 2006; Riaño, 2006; Comisión Nacional de Reparación y Reconciliación, 2009; Nieto, 2010; Organización de estados americanos, 2011; Duque \& Gordon, 2012; Duque \& Pineda, 2013; Vidales, 2013; Villa, 2014). Esta es presentada como una estrategia para contribuir al fortalecimiento de la identidad cultural de un pueblo. La memoria colectiva consiste en "la producción narrativa de la historia; siempre produce una selección de acontecimientos del pasado en relación con el presente, que son organizados de acuerdo con significados cada vez actualizados" (Santamaria y Marinas 1995 citado por Molina, 2010, p. 67). De esta manera, se proponen los ejercicios de memoria colectiva como una posibilidad superar las situaciones de victimización al lograr una reconstrucción de la historia dolorosa que se vivió antes, durante y después de los hechos de violencia.

Las propuestas de construcción de memoria colectiva toman en consideración la categoría de la identidad como un eje importante para el acompañamiento. Desde estas perspectivas, la identidad es entendida como un proceso en constante construcción a partir de las diferentes relaciones que se entablen en el curso vital de un individuo (Estrada y otros, s.f.). En este orden de ideas, Arévalo (2010) llama la atención sobre el papel que juega el contexto político como un ámbito de relación que incide en el proceso de construcción de la identidad. De esta manera, al identificar las diferentes relaciones establecidas con representantes de las instituciones y la comunidad, se pueden rastrear las transformaciones que ha sufrido la identidad gracias a los discursos y prácticas con los que han interactuado. Esto contribuye a la transformación de la parálisis, el miedo generalizado o la desesperanza y pasar al movimiento, la renovación de la confianza en el otro, la esperanza en que sus sueños se pueden hacer realidad (Estrada, y otros, S.f). En este sentido, es necesario trabajar en las intervenciones aspectos que apunten a tocar los elementos constitutivos de la identidad que son los que se han visto resquebrajados con la incursión violenta.

Una forma a través de la cual se aborda la ruptura de los referentes sociales de la vida comunitaria -expresados en espacios que cotidianamente permitian compartir, divertirse, aprender y que pasan a ser inhabitados cuando exactamente alli es donde se han cometido las masacres, que se fracturan después de un hecho violento- es la memoria histórica. Es por esta razón que muchas de las intervenciones dirigidas a trabajar la recuperación de la memoria histórica van dirigidas al rescate de dichos espacios tan importantes en la vida de una comunidad, porque es precisamente alli donde construyen lazos sociales, se fortalecen, se apoyan, sueñan, se comprometen y crecen como grupo humano. 


\section{La reivindicación del sujeto en los procesos de atención.}

Pensar el ámbito de la atención psicosocial a víctimas implica considerar que el reconocimiento de un sujeto bajo el estatuto de víctima del conflicto armado es justificable desde la perspectiva de los derechos humanos y la defensa de la dignidad de las personas sometidas a las acciones violentas. No obstante, dicho reconocimiento implica el riesgo de que la clasificación como víctima sea llevada al ámbito de la subjetividad (Moreno, 2013). Desde esta perspectiva, la revisión documental en torno a los procesos de atención psicosocial a víctimas se ve enriquecida por reflexiones que apuntan a la reivindicación del sujeto en los procesos de atención. A partir de dichas reflexiones es posible argumentar que la categoría de víctima es indicativa de un estatuto jurídico necesario, sin embargo, es importante considerar que el significante víctima tiene una carga valorativa que está asociada a los efectos que trae para un sujeto el sometimiento a los actos de violencia, de modo que cuando se clasifica a los sujetos como víctimas existe el riesgo de que sean entendidos como representantes de una categoría predefinida y por ello se implementen soluciones estandarizadas para su tratamiento.

Algunos autores (Sánchez \& Musitu, 1996; Martín-Beristain, 2004; Meertens, 2006; Estrada $\&$ otros, s.f.) argumentan que la forma como se rotula a las personas que han vivenciado un hecho de violencia producto del conflicto armado va a marcar el derrotero de la intervención que se realice, así como también la forma como los profesionales se relacionan con los destinatarios de su atención. Esto se hace visible en las interacciones que establecen los actores en los escenarios de atención psicosocial, y se traduce en aquello que los agentes de la atención demandan a los destinatarios de los servicios, es decir, el tipo de actividades que se proponen y la forma cómo se agencian las mismas. Por ejemplo, es preciso observar si los programas de asistencia favorecen la capacidad de elección de sus destinatarios, y si en esa medida invitan a asumir la responsabilidad frente las elecciones o por el contrario se impone todo y se promueve una actitud pasiva, orientada a esperar a que otro resuelva las angustias del sujeto.

En este mismo orden de ideas, Martin-Beristain (2004) plantea que "frecuentemente toda la interacción o las formas de ayuda humanitaria o las formas de atención psicosocial están mediatizadas por la experiencia vista como un estigma, esto es muy negativo para la víctima y para la relación de ayuda" (p. 37). Lo anterior encuentra un ejemplo en los rótulos bajo los cuales han sido nombrados las personas en situación de desplazamiento por parte de las diferentes instituciones que los atienden y en ocasiones por los investigadores. Dichos rótulos generan una redefinición en su identidad, con el agravante de que algunos de ellos los definen como no confiables y pasivos, lo cual dista en la mayoría de los casos de la realidad, ya que en sus lugares de origen eran reconocidos como trabajadores honrados, en oposición a la idea degradante que se ha popularizado en las ciudades, que los ubica en competencia por las oportunidades de los habitantes de las zonas urbanas y también como sujetos pasivos a la espera de que otro resuelva su vida. En contraste con ello, se propone como alternativa para combatir dicha estigmatización, el rescate de las estrategias de supervivencia que dan cuenta del coraje y valor con el que se sobreponen a las experiencias de horror por las que han pasado las personas en situación de desplazamiento (Meertens, 2006). 
Estrada y otros (S.f), así como Meertens (2006), hacen énfasis en la contradicción existente en la categoría víctima, pues sugiere desvalimiento y pasividad, mientras que algunas experiencias de trabajo con personas afectadas por hechos de violencia dan cuenta de la posibilidad de agencia, capacidad de resolución y afrontamiento ante las adversidades. No obstante, como plantea Jaramillo (2006), el rótulo de víctima es el que favorece el reconocimiento por parte del Estado y con ello la posibilidad de recibir la asistencia que éste debe brindar de acuerdo con la ley.

En este contexto, autores como Velásquez (2008) advierten del riesgo que implica las relaciones establecidas entre el sujeto y el otro durante los procesos de atención, ya que existe el peligro de que ambos queden instalados en identificaciones caprichosas (víctima / benefactor), de manera que el sujeto puede inscribirse en estas identificaciones como una estrategia inconsciente para huir de la dificultad de ser, impidiendo que se implique de manera responsable en su proceso. En este sentido se encuentran dos extremos posibles, uno en el que la identificación como víctima no aporta una exigencia a futuro y otro en el que la identificación permita una exigencia a futuro para que los sujetos se impliquen como actores responsables en la construcción del mismo. En el primer caso se trata de una identificación que niega la subjetividad y en el segundo, una que posibilita la emergencia de la misma. Es así como Castro (2002) sugiere que el trabajo con víctimas se oriente a acompañar, desencadenar y coadyuvar en su proceso de subjetivación buscando de esta forma la exigencia de futuro frente al hecho de victimización y establecerse como actores responsables de su devenir.

Desde esta misma óptica, algunos autores se oponen a la rotulación que ubica a la persona como un desplazado o una víctima centrándose solamente en su situación en el momento en el que se presenta como afectado por un hecho de violencia y dejando de lado lo que esa persona era antes de que ocurriera dicho evento (Mertens, 2006). Otros coinciden con esta perspectiva al plantear que la nominación legal de víctima comporta el peligro de que no se defina como una situación de paso, sino que se convierta en la oportunidad de hacerse a un nombre propio, de manera que los programas de atención se encontrarán con beneficiarios que se perpetuarán en esta condición y en la crítica a lo que reciben, dado que ninguna ayuda les será suficiente (Gallo, 2008). Esto se relaciona con el hecho de que las acciones dirigidas a la población afectada por hechos de victimización se enmarcan en una política de Estado asistencialista que refuerza la actitud de mendicidad y de dependencia en la población (Aguilera, 2010).

En esta misma vía, Gallo (2008) cuestiona los programas de atención a víctimas del conflicto que se conforman con la orientación jurídica en cuestión de derechos y deberes y con prestar asistencia social, señalando que este tipo de programas no trabajan en la vía de devolver al sujeto la dignidad de su palabra. En este sentido, en vez de atender a las personas como una cifra, recomienda escucharles con la intención de ayudar a producir una resignificación histórica lo vivido. Se hace evidente entonces la preocupación por cómo realizar una intervención con personas afectadas por el conflicto armado, poniendo en primer plano la subjetividad. Ante esta cuestión Jaramillo (2006) propone que la noción de víctima y su traducción en cifras supone una dificultad en tanto que introduce el anonimato, el borramiento de la particularidad del sufrimiento, por lo tanto aboga por la 
transformación de la cifra estadística en un sujeto que sufre de manera singular, ya que los hechos violentos se inscriben en los sujetos de formas distintas.

Villa e Insuasty (2015) manifiestan en su investigación sobre reparación en San Carlos Antioquia, que "se evidencia entonces que el proceso de reparación es complejo en la medida en que no puede aparecer como una fórmula copiada y aplicada al pie de la letra, es necesario, además, que este se haga dentro de una lectura del contexto, responsable, profunda y compleja, que considere a los actores protagonistas (las víctimas y las comunidades azotadas por el conflicto), las temporalidades y las necesidades propias de la gente, apuntando realmente a la satisfacción de las mismas". (Villa \& Insuasty Rodriguez, 2015).

En este orden de ideas, es preciso reconocer que las afectaciones subjetivas hacen referencia al reconocimiento, en cada sujeto, de un universo de sentidos y significaciones configurados a través de su devenir personal y su relación dialéctica con los otros, a partir de los cuales se organiza su realidad psíquica. En consecuencia con ello, las formas de estar en el mundo, las relaciones con otros, los modos de satisfacción, las maneras de vivir el bienestar y padecer el malestar, tienen un carácter subjetivo. De esta manera, se asume que los impactos psicosociales en los sujetos y poblaciones afectados por hechos de victimización en el marco del conflicto armado, comprenden el análisis de aspectos singulares, culturales, sociales, económicos y políticos que cuentan con un carácter estructural, así como también factores asociados a la pertenencia étnica, los grupos de edad, el género, la orientación sexual y la discapacidad, entre otros, que configuran el conjunto de variables en las que se sitúa la complejidad de la realidad humana.

Se señala entonces una oposición entre víctima y sujeto, y es al campo del segundo al que se dirige la intervención de los profesionales en los escenarios de atención psicosocial. Desde esta perspectiva la forma en la que se comprende el objeto de intervención es clave para establecer los lineamientos de la atención psicosocial. En este sentido, si la intervención está enfocada a víctimas se limitará la actuación de los participantes, quienes estarán siempre pasivos frente a su situación. Mientras que una comprensión de los participantes como sujetos que reconozca su historia, sus tradiciones y sus costumbres, permite una escucha de éstos y les otorga la responsabilidad en su proceso de reparación y restablecimiento de su ciudadanía (Jaramillo, 2006).

Reconocer esta situación invita a trascender la categoría jurídica de víctima, en la medida en que dicha denominación se puede convertir en un referente de identificación desde el cual el sujeto hace lazo social. En su lugar, podrian intentarse fórmulas como sujetos afectados por hechos de victimización, la cual hace alusión a la victimización como un acontecimiento más que como una condición. De esta manera, la victimización es entendida como un evento que se suma a la cadena de acontecimientos que constituyen la historia de un sujeto, una familia o una comunidad. El orden del acontecimiento es diferente al orden de la identificación: la identificación implica asumir la categoría como si fuera un rasgo determinante, mientras que el orden del acontecimiento brinda la posibilidad de resignificar lo sucedido a partir de la historización (Moreno \& Moncayo, 2015). 


\section{Acuerdos sobre una postura prescrita.}

El análisis de las posturas sugeridas para la atención psicosocial a víctimas del conflicto armado en Colombia da cuenta, de manera general, de la preponderancia de marcos de referencia que se ubican desde una perspectiva crítica frente al discurso de la salud mental y la clasificación psicopatológica, avanzando hacía perspectivas que se fundamentan en la psicología social y la psicología comunitaria, desarrolladas principalmente en contextos latinoamericanos. Tanto en las en las reflexiones investigativas como en los análisis de las experiencias de atención es común encontrar que se rescaten principios tales como la necesidad de hacer un análisis contextualizado de las situaciones de violencia y los efectos que pueden generar en las comunidades y sujetos. Así mismo, hay un marcado énfasis en una apuesta por el reconocimiento y la dignificación, como también por el empoderamiento de los sujetos afectados por los hechos de victimización. Ello supone una noción de sujeto capaz de construir, a partir de sus propios recursos, las formas de hacer frente a las dificultades que ha tenido que enfrentar como consecuencia de la violencia. Las posturas presentadas están orientadas a la acción sin daño e insisten en la necesidad de cuidar el rol de los representantes de las instituciones que brindan atención, de tal forma que las acciones contribuyan a la reparación, respetando los principios de dignificación y reconocimiento.

La postura oficial del Estado coincide con estos planteamientos y los ha formalizado en sus documentos de difusión y capacitación, lo que da cuenta de un trabajo reflexivo y de la incorporación de los análisis y los resultados de las investigaciones adelantadas en cuanto a esta materia. De esta manera, esta revisión hace pensar que respecto a la atención psicosocial a víctimas del conflicto armado podemos hablar de un acuerdo en una postura prescrita. Este es un gran avance para el proceso de reparación integral a las víctimas y además es un indicador de la experiencia acumulada durante el desarrollo de dicho proceso.

No obstante, es preciso advertir que la postura prescrita no garantiza que las acciones que se llevan a cabo día a día en los escenarios de atención respondan de manera pura al ideal sugerido. Esto se debe a que entre los agentes de la intervención y los sujetos afectados por los hechos de victimización tiene lugar una relación intersubjetiva. De esa manera, los efectos de las acciones de respuesta promovidas por las intervenciones psicosociales son contingentes e instalados en el campo de las relaciones humanas entre sujetos de lenguaje, por ello son del orden de lo impredecible (indecible); es decir, no se pueden calcular con precisión. Así las cosas, es difícil pensar en una forma totalmente preestablecida cuando se trata de un programa de intervención. En los procesos de atención psicosocial es posible trazar una estrategia que se oriente al cumplimiento de objetivos específicos, pero en el curso de las relaciones intersubjetivas allí implicadas se pone en juego lo inconmensurable. Lo inconsciente acude a la cita y pone en marcha otra escena de relaciones que escapan al control de las voluntades particulares de los actores y sus intenciones imaginarias (Moreno \& Moncayo, 2015).

Por esta razón, las investigaciones deben avanzar en el análisis de los escenarios de atención y las relaciones establecidas entre los actores que participan del proceso, así como también en la observación de la implementación de las prácticas de atención psicosocial y el rol asumido por los profesionales que asumen esta labor. A su vez, es necesario implementar estrategias para el análisis de los efectos de los programas que, más allá de la 
lógica de la fiscalización y vigilancia con fines de rendición de cuentas, estén orientadas a la recuperación de las experiencias de atención y la manera en que las mismas son asumidas por los sujetos a quienes están dirigidas, con el objetivo de identificar buenas prácticas y aspectos a fortalecer.

\section{Referencias bibliográficas.}

Aguilera, A. (2010). El silencio impuesto a la recuperación de la memoria: una propuesta de recuperación psicológica a víctimas de violencia sociopolítica en Colombia . En J. Salas J, Memoria, silencio y acción psicosocial. Reflexiones sobre porque recordar en Colombia (págs. 159-178). Bogotá: Cátedra Libre.

Ardila, S., Páez, J., Peinado, J., \& Prieto, E. (2002). Los niños como actores en la construcción de tejido social: una oportunidad de aprendizaje a partir de la experiencia en altos de Cazucá. En M. N. Bello, \& S. Ruíz Ceballos, Conflicto armado, niñez y juventud. Una perspectuva psicosocial (págs. 350-352). Bogotá: Universidad Nacional de Colombia.

Arévalo, L. (2010). Atención y reparación psicosocial en contextos de violencia socio política: una mirada reflexiva. Revistas de estudios sociales(36), 29-39.

Arias, J., Arévalo, L., \& Ruíz, S. (2002). Educación y conflicto armado . En M. N. Bello , \& S. Ruíz Ceballos , Conflicto armado, Niñez y Juventud. Una perspectiva psicosocial (págs. 179-277). Bogotá: Universidad Nacional de Colombia .

Bello, M. N. (2006). Enfoque psicosocial. En M. N. Bello, Convenio interadministrativo $N^{\circ} 306$ de 2005. Evaluación y estudio técnico de la gerencia e impacto social de los proyectos de intervención psicosocial a la población en situación de desplazamiento forzado interno por la violencia en Colombia. 2000 - 2005 (págs. 34-44). Bogotá: Universidad Nacional de Colombia.

Bello, M. N. (2006). Implicaciones éticas y metodológicas de la investigación contratada. En M. N. Bello, Investigación y desplazamiento forzado. Reflexiones éticas y metodológicas (págs. 45-56). Bogotá: Red nacional sobre el desplazamiento forzado.

Bello, M. N. (2006b). Investigaciones éticas y metodológicas de la investigación contratada. En M. N. Bello, Investigación y desplazamiento forzado. Reflexiones éticas y metodológicas. (págs. 45 - 56). Bogotá: Red nacional sobre el desplazamiento forzado en Colombia. Colciencias.

Castro, M. C. (2002). Jóvenes guerreros: elecciones, pasajes y pasos. En M. N. Bello, \& S. Ruíz Ceballos, Conflicto armado niñez y juventud. Una perspectiva psicosocial (págs. 7090). Bogotá: Universidad Nacional de Colombia.

Comisión Nacional de Reparación y Reconciliación. (2009). Memorias en tiempo de guerra. Repertorio de iniciativas. Bogotá: Punto aparte Editores.

Congreso de Colombia. (2012). Ley de víctimas y restitución de tierras y sus decretos reglamentarios. Bogotá: Imprenta nacional de Colombia. 
Díaz , M., Arias , N., \& Lasso, P. (2010). De la metodología a la metódica: Peregrinajes imperiosos en la investigación social. Cali: Editorial Bonaventuriana.

Díaz, M. R., Blandón, L. M., \& Echeverri, G. (2006). Memoria y cotidianidad: Herramientas ético-metodológicas para restaración de la medicina tradicional en grupos humano en desplazamiento forzado. En M. N. Bello, Investigación y desplazamientoforzado. Reflexiones éticas y metodológicas (págs. 151-159). Bogotá: Red nacional de desplazamiento forzado en Colombia.

Duque, N., \& Gordon, D. (2012). Acompañamiento comunitario a poblaciones víctima del conflicto armado. Una propuesta de memoria histórica a partir de la fotografía. Cali: Editorial Bonaventuriana.

Duque, N., \& Pineda, J. (2013). El conflicto armado en el pacífico colombiano. El caso Sabaletas 2013. En A. Castillejo Cuellar, \& F. Reyes Albarr, Memoria, violencia y sociedad. Debates y agendas en la Colombia actual (págs. 541-555). Bogotá: Ediciones Usta. Universidad Santo Tomás.

Durán, E. (2002). Elementos para un análisis de las politicas de atención a la niñez y la juventud víctimas del conflicto armado. En M. N. Bello, \& S. Ruíz Ceballos, Conflicto armado niñez y juventud. Una perspectiva psicosocial (págs. 169-177). Bogotá: Universidad Nacional de Colombia.

Estrada, Á., Ripoll , K., Rodríguez, D., Antholin, N., Céspedes, M. P., \& Acuña, M. (s.f.). Psicología social critica: Estrategias para la atención del impacto psicosocial acumulado en jóvenes, y sus redes sociales, afectados por el conflicto interno en Colombia. Bogotá: Universidad de los Andes- Centro internacional de investigaciones para el desarrollo IDRC (Canadá.

Estrada, Á., Ibarra , C., \& Sarmiento , E. (2003). Regulación y control de la subjetividad y la vida en el contexto del conflicto armado colombiano. Revista de Estudios Sociales(15), $133-149$.

Estrada, A., Ripoll, K., \& Rodríguez, D. (2010). Intervención psicosocial con fines de reparación con víctimas y sus familias afectadas por el conflicto interno en Colombia: Equipos psicosociales en contextos jurídicos. Revista de Estudios Sociales(36), 239-247.

Gallo, H. (2008). Subjetividad y conflicto armado. En N. E. Medellín, Conflicto armado: memoria, trauma y subjetividades (págs. 37-40). Medellin: La carreta Editores.

Grupo de Jóvenes afrocolombianos - Taller vida. (2002). Jóvenes afrocolombianos constructores de paz. En M. N. Bello , \& S. Ruíz Ceballos, Conflicto armado niñez y juventud. Una perspectiva psicosocial (págs. 336-349). Bogotá: Universidad Nacional de Colombia.

Grupo de psicología social crítica . (2010). Del dolor a la propuesta. Voces del panel de víctimas. Revista de Estudios Sociales(36), 114-125. 
Grupo de psicología social crítica. (2010). Principios éticos para la atención psicosocial. Revista de Estudios Sociales(36), 128-131.

Investigación reciente sobre la violencia en Colombia: Un contexto para la política pública sobre desplazamiento forzado. (2006). En M. N. Bello , Plata, J. J. (2006). Investigación y desplazamiento forzado. Reflexiones éticas y metodológicas (págs. 17-30). Bogotá: Red nacional sobre desplazamiento forzado en Colombia.

Jaramillo, J. (2006). Reubicación y restablecimiento en la ciudad. Estudio de caso con población de desplazamiento. Universitas Humanísticas, 62(2), 143-168.

Lasso, P. (2013). Cuando se vive el desarraigo. Educación y desplazamiento forzado: Una mirada desde el distrito de Aguablanca, Cali, Colombia. Revista científica Guillermo de Ockham, 11(2), 35-51.

Marin, G. (1996). Consideraciones necesarias en el diseño culturalmente apropiadas en la promoción de la salud. En C. San Juan Guillen, Intervención psicosocial. Elementos de programación y evaluación socialmente eficaces (págs. 36 - 45). Barcelona: Antrhopos.

Marín, G. (1996). Consideraciones necesarias en el diseño culturalmente apropiadas en la promoción de la salud. En S. Guillen, Intervención psicosocial. Elementos de programación y evaluación socialmente eficaces (págs. 36-45). Barcelona: Antrhopos.

Martín Baró, I. (1985). Acción e ideología: psicología social desde Centroamérica I. San Salvador: UCA.

Martín-Beristain , C. (2004). Enfoques y metodologias de atención psicosocial en el contexto del conflicto sociopoliticos. Bogotá: Terre des Hommes-Italia.

Martín-Beristain, C. (2012). Acompañar los procesos con las víctimas. Colombia: Programa promoción de la convivencia.

Mc. Cauley, U. (2002). Conflicto armado, niñez y juventud, los fenómenos globales. En M. N. Bello , \& S. Ruíz Ceballos, Conflicto armado niñez y juventud. Una perspectiva psicosocial (págs. 379-394). Bogotá: Universidad Nacional de Colombia.

Meertens, D. (2006). Reflexiones éticas, metodológicas y conceptuales sobre la investigación en desplazamiento y género. En M. N. Bello, Investigación y desplazamiento forzado. Reflexiones éticas y metodológicas (págs. 112 - 125). Bogotá: Red nacional sobre desplazamiento forzado en Colombia. Colciencias.

Meertens, D. (2006). Reflexiones éticas, metodológicas y conceptuales sobre la investigación en desplazamiento y género. En M. N. Bello, Investigación y desplazamiento forzado. Reflexiones éticas y metodológicas (págs. 112-125). Bogotá: Red nacional sobre desplazamiento forzado en Colombia. 
Ministerio de salud y protección social. (2013). Programa de atención psicosocial y salud integral a víctimas - Papsivi. Obtenido de www.minsalud.gov.co: https://www.minsalud. gov.co/proteccionsocial/Paginas/Victimas_PAPSIVI.aspx

Ministerio de salud y protección social. (2013). Protocolo de atención integral en salud con enfoque psicosocial para las personas víctimas del conflicto armado en Colombia. Bogotá: Ministerio de salud y protección social.

Molina, N. (2006). Psicología politica, resistencia y democracia: la resistencia comunitaria y la transformación de conflictos. Buenos Aires: Editorial Proa.

Molina, N. (2010). Reconstrucción de la memoria en historias de vida. Efectos políticos y terapéuticos. Revista de Estudios Sociales, 64-75.

Moreno, M. (2013). Psicoanálisis e intervención social. Revista CS, 115-142.

Moreno, M., \& Moncayo, J. (2015). Abordaje psicosocial: Consideraciones conceptuales y alternativas de análisis en el escenario de atención a víctimas del conflicto armado. En E. Moncayo , \& Á. Díaz, Psicología social critica e intervención psicosocial: Reflexiones desde la investigación. Cali: Editorial Bonaventuriana.

Nieto, P. (2010). Relatos autobiográficos de víctimas del conflicto armado: una propuesta metodológica. Revista de estudios sociales, 76-85.

Novoa, M. (2013). Reflections of the psychological damage of people exposed to war situations in Colombia. En S. Sacipa , \& M. Montero, Psychosocial Approaches to peace-building (págs. 41-57). Bogotá: Springer.

Organización de los estados americanos. Misión de apoyo al proceso de paz Colombia . (2011). Identidad e imágenes. Sabaletas, un pueblo con memoria. Cali: Equilibrio gráfico editorial.

Osorio, F. E. (2006). "Dime con quién andas y te diré de qué lado estás". Relaciones, alianzas e investigación social en contextos de guerra. En M. N. Bello, Investigaciones y desplazamiento forzado. Reflexiones éticas y metodológicas (págs. 31-45). Bogotá: Red nacional sobre desplazamiento forzado en Colombia.

Palacio , J., \& Sabatier, C. (2002). Impacto psicológico de la violencia en Colombia: salud mental y redes sociales en familias desplazadas en el caribe. Barranquilla: Uninorte.

Rebolledo, O., \& Rondón , L. (2010). Reflexiones y aproximaciones al trabajo psicosocial con víctimas individuales y colectivas en el marco del proceso de reparación. Revista de Estudios Sociales, 40-50.

Riaño, P. (2006). El desplazamiento interno y los trabajos de la memoria. En M. N. Bello, Investigación y desplazamiento forzado. Reflexiones éticas y metodológicas (págs. 99111). Bogotá: Red nacional sobre desplazamiento forzado en Colombia. 
Ruíz, S. (2002). Impactos psicosociales de la participación de niñ@s y jóvenes en el conflicto armado. En M. N. Bello , \& S. Ruíz Ceballos, Conflicto armado niñez y juventud. Una perspectiva psicosocial (págs. 17-46). Bogotá: Universidad Nacional de Colombia.

Sacipa, S. (2013). To feel and to re-signify forced displacement in Colombia. En S. Sacipa, \& M. Montero, Psychosocial Approaches to peace-building (págs. 59-74). Bogotá: Springer.

San Juan Guillén, C. (1996). Intervención Psicosocial: del individuo a la comunidad. En C. San Juan Guillén, Intervención psicosocial: elementos de programación y evaluación socialmente eficaces (págs. 46-49). Barcelona: Anthropos.

Sánchez , A., \& Musitu, G. (1996). Cuestiones éticas en la intervención psicosocial. En G. Sánchez Vidal , \& M. Ochoa, Intervención comunitaria: Aspectos científicos, técnicos y valorativos (págs. 79-85). Barcelona: EUB.

Toro, D. M. (2011). Acompañamiento psicosocial a niños y niñas víctimas del conflicto armado en Colombia: Un escenario para a acción sin daño. Tesis imédita de Especialización. Bogotá: Universidad Nacional de Colombia. Obtenido de http://bivipas.info/ bitstream/10720/570/1/TT-134-Toro_Diana-2011-439.pdf

Tovar, C. (2013). Personal resources and empowerment in a psychosocial accompaniment process. En S. Sacipa, \& M. Montero, Psychosocial Approaches to peace-building (págs. 75-88). Bogotá: Springer.

Unidad para la atención y reparación integral a las víctimas. (2014). lementos para la incorporación del enfoque psicosocial en la atención, asistencia y reparación a las víctimas. Obtenido de www.unidadvictimas.gov.co/: http://www.unidadvictimas.gov.co/index. $\mathrm{php} /$ sala-de-prensa/biblioteca-virtual

Velásquez, J. F. (2008). Advertencias para el trabajo bajo la transferencia con sujetos afectados por la violencia. En Nueva Escuela Lacaniana de Medellín, Conflicto armado: memoria, trauma y subjetividad (págs. 135-142). Medellín: La Carreta Editores.

Vidales, R. (2013). Memory, narrative and the social transformation of reality. En S. Sacipa , \& M. Montero, Psychosocial Approaches to peace-building (págs. 89-110). Bogotá: Springer.

Villa, J. D. (2012). La acción y el enfoque psicosocial de la intervención en contextos sociales: Podemos pasar de la moda a la precisión teórica, epistemológica y metodológica? . $\mathrm{El}$ Agora USB, 12(2), 349-365.

Villa, J. D. (2014). Memoria, historias de vida y papel de la escucha en la transformación subjetiva de víctimas / sobrevivientes del conflicto armado colombiano. El Agora USB, 14(1), 37-60.

Villa, J. D., \& Insuasty Rodriguez, A. (2015). Significados en torno a la reparación, la ayuda humanitaria, la indemnización y la restitución en víctimas del conflicto armado en el municipio de San Carlos. El Agora USB, 15(2), 419-445. 
Wilches, I. (2010). Lo que hemos aprendido sobre la atención a mujeres víctimas de violencia sexual en el conflicto armado colombiano. Revista de Estudios Sociales, 86-94.

\section{Nota:}

Este articulo fue realizado

${ }^{3}$ Este artículo fue realizado en el macro de la investigación Posturas y prospectiva en el abordaje psicosocial de población víctima de la violencia por el conflicto armado, desarrollada en el grupo de investigación estéticas urbanas y socialidades de la Universidad San Buenaventura Cali. 